Aim of the study: Actual lymphatic drainage of pancreatic body neoplasms and the proper extent of lymphadenectomy remain unknown. The aim of the study was to define the exact lymphatic draining pattern using the dye mapping method.

Material and methods: The study enrolled patients who were operated on for tumor of the pancreatic body in the Department of General and Transplant Surgery of the Medical University of Lodz during 2010, with injection of $1 \mathrm{ml}$ of blue dye (Patent Blue, Guerbet) in the centre of the neoplasm and sentinel node identification. Radical surgical management included distal pancreatectomy, whereas gastrojejunal or triple bypass anastomoses were performed in irresectable cases.

Results: The study group consisted of 13 patients with locally advanced tumors of the pancreatic body (T3 and T4, mean tumor size $4.9 \mathrm{~cm}$ ). Lymphatic mapping was able to identify sentinel nodes in 5 of 13 cases (38.46\%). A sentinel node was found in station 11p ( 3 cases) and 9 ( 1 case). Skip metastasis to the left gastric artery node (group 7) was noted. All identified sentinel nodes were metastatic; tumor deposits were confirmed in non-sentinel nodes as well.

Conclusions: In advanced pancreatic body tumors feasibility of sentinel node navigation is considerably restricted. Further studies in smaller tumors using optimized newer markers may define the exact lymphatic draining pattern.

Key words: pancreatic body tumor, sentinel node, lymphadenectomy.

\section{Sentinel lymph node mapping in tumors of the pancreatic body: preliminary report}

\author{
Adam Durczyński ${ }^{1}$, Piotr Hogendorf ${ }^{1}$, Dariusz Szymański ${ }^{1}$, Piotr Grzelak², \\ Janusz Strzelczyk ${ }^{1}$
}

${ }^{1}$ Department of General and Transplant Surgery, Medical University of Lodz, Barlicki University Hospital, Lodz, Poland

2Department of Radiology and Diagnostic Imaging, Medical University of Lodz, Lodz, Poland

\section{Introduction}

Surgical excision of pancreatic cancer remains the gold standard of treatment [1]. Nevertheless, 5-year postoperative survival rates of these patients are unsatisfactory [2], due to high incidence of lymphatic invasion and poor loco-regional cure [3].

So far, the application of sentinel node biopsy in gastrointestinal cancers, initially introduced in malignant melanoma $[4,5]$ and breast cancer $[5,6]$ and based on the orderly progression of cancer cells within the lymphatic system, has confirmed multidirectional drainage. Several studies indicate occurrence of skip metastases beyond regional lymph nodes, resulting in inadequate staging of gastrointestinal cancers [7], and extensive lymphadenectomy according to the Japanese classification is recommended to improve survival in these patients [8]. Currently, in cancer of the pancreatic head, standard lymphadenectomy includes lymph nodes located peripancreatically as well as to the right of the superior mesenteric artery and along the proper hepatic artery. In contrast, studies concerning the extent of lymphadenectomy in cancers of the pancreatic body hardly exist, while their occurrence rate is about 25\% [9], and the actual lymphatic drainage of these neoplasms and proper extent of lymphadenectomy remain unknown. Lymphatic mapping seems to be an essential tool for identifying complicated lymphatic streams in pancreatic cancer in order to plan an adequate extent of surgical resection $[4,10,11]$. Thus, the aim of our study was to define the exact lymphatic draining pattern from pancreatic body tumors using the dye lymphatic mapping method.

\section{Material and methods}

This study enrolled patients hospitalized in the Department of General and Transplant Surgery of the Medical University of Lodz during 2010, who were operated on for tumor of the pancreatic body with preoperative view of radical operation. Preoperative aspiration tumor biopsy confirming cancer was not applied. Subjects with distant metastases were excluded preoperatively. No exclusions were made based on intraoperative irresectability. Tumor size was recorded for all patients.

At laparotomy, the lesser sac was opened through an incision into the gastrocolic ligament to visualize the tumor of the pancreatic body. Before mobilization of the pancreas for resection $1 \mathrm{ml}$ of blue dye (Patent Blue, Guerbet) was injected in the centre of the tumor. After 5 minutes, lymphatic drainage routes revealed by colored lines were assessed with great care and separate biopsy of blue-dyed lymph node was performed. Since dye migration time to the first lymph node was short, identification with optional lymph node biopsy had to be instant, since 
Table 1. Location of sentinel node in individual subjects. The mean tumor size was $4.9 \mathrm{~cm}(\mathrm{range}, 3-7 \mathrm{~cm})$

\begin{tabular}{|c|c|c|c|}
\hline No. & Diagnosis & Operation procedure & Blue-dyed sentinel node \\
\hline 1 & adenocarcinoma & distal pancreatectomy & solitary in station 7 \\
\hline 2 & adenocarcinoma & gastroenteroanastomosis & solitary in station $11 \mathrm{p}$ \\
\hline 3 & adenocarcinoma & distal pancreatectomy & solitary in station $11 \mathrm{p}$ \\
\hline 4 & adenocarcinoma & triple bypass & - \\
\hline 5 & adenocarcinoma & triple bypass & - \\
\hline 6 & adenocarcinoma & triple bypass & - \\
\hline 7 & adenocarcinoma & triple bypass & - \\
\hline 8 & adenocarcinoma & triple bypass & - \\
\hline 9 & adenocarcinoma & distal pancreatectomy & - \\
\hline 10 & adenocarcinoma & distal pancreatectomy & - \\
\hline 11 & adenocarcinoma & gastroenteroanastomosis & $\begin{array}{l}\text { two routes; blue node in station } 9 \\
\text { and negative in station } 14 a\end{array}$ \\
\hline 12 & adenocarcinoma & triple bypass & - \\
\hline 13 & adenocarcinoma & gastroenteroanastomosis & solitary in station $11 \mathrm{p}$ \\
\hline
\end{tabular}

otherwise further lymph node stations might have been bluedyed. However, rapid and traumatic preparation had to be avoided, since it might have injured proximal lymphatic channels leading to the sentinel node, with dye flow to surrounding tissues and impossible sentinel node identification.

Blue (sentinel) lymph nodes were sent for further histopathological examination. In resectable tumors surgical management included radical distal pancreatectomy with excision of regional lymph nodes. In irresectable cases gastrojejunal or triple bypass anastomoses were performed, and specimens of pancreatic tumor and enlarged (non-sentinel) lymph nodes were sent for further histopathological evaluation.

\section{Results}

The study group consisted of 13 patients (mean age $50 \pm 9, M / F$ ratio $4: 1$ ), after written informed consent, with locally advanced tumors of the pancreatic body (T3 and T4 according to the TNM scale). Preoperative imaging studies excluded distant metastases, but mostly failed in evaluation of tumor resectability and lymph node involvement in comparison to the intraoperative view.

Lymphatic mapping was able to identify sentinel nodes in the minority of cases with a positive rate of sentinel node detection of $38.46 \%$ (5/13 of cases); final diagnosis, type of operation, mean tumor size and blue-dyed sentinel nodes are summarized in Table 1. Most commonly, the sentinel node was localized along the proximal splenic artery (3 cases; group 11p according to Japanese classification) [5]. In one case, skip metastasis to the left gastric artery node (group 7) was noted. All identified sentinel nodes were metastatic, although tumor deposits were confirmed in other lymph nodes of these patients.

\section{Discussion}

Sentinel node biopsy is standard procedure among patients with malignant melanoma [7, 8] and breast cancer
$[8,9]$. Due to the evident benefits of lymphatic mapping in these malignancies, this technique has been recently used in cases of solid tumors of the gastrointestinal tract including the pancreas [10-13]. Intraoperative, selective sentinel node biopsy is considered to be a revolutionary surgical management of these patients. Some authors recognize this method as an essential tool for identifying complicated lymphatic streams from upper gastrointestinal cancers and distribution of skip metastases, which pass the closest station of lymph nodes, according to the Japanese classification [5], and may not be taken during standard lymphadenectomy [14]. Various studies indicate that skip metastases may occur among 9 to $19 \%$ of all patients with a solitary lymph node metastasis [4]. Detection and accurate investigation of this node may rule out understaging of gastrointestinal cancers, which is considered as a phenomenon responsible for poor loco-regional control and chemoresistance of upper gastrointestinal cancers. Unfortunately, our study failed to detect the actual pattern of lymph flow from tumors of the pancreatic body, for several reasons. Firstly the natural course of pancreatic body tumors is mostly asymptomatic, which makes its detection delayed, and they are usually diagnosed in a late stage of local advancement, as suggested by our study. Thus, in this stage of tumor lymphatic channel invasion and its occlusion is highly possible. Early metastatic spread with massive lymph node involvement may divert or obscure lymph flow, which prevents sentinel node identification [15]. The negative correlation between sentinel node detection rate and tumor advancement in breast cancer is well known [16] and pancreatic body tumor size in our group was bigger than breast cancer usually evaluated by lymphatic mapping [17]. Additionally, surgeon's experience in sentinel node identification techniques was proved as an independent factor improving its detection, with a learning curve of more than 20 patients in the case of breast cancer [18]. In our study group, operations were performed by high-volume 


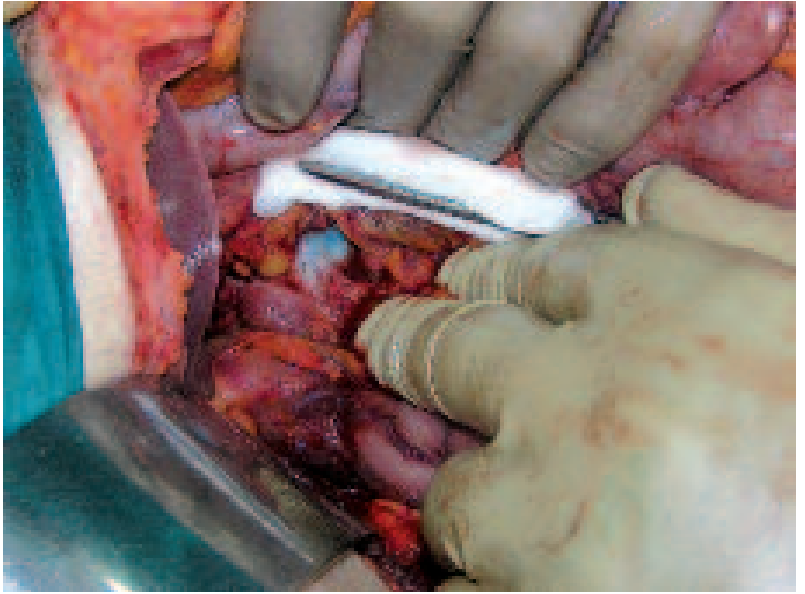

Fig. 1. Solitary sentinel lymph node of the celiac axis group

surgeons performing approximately 100 pancreatic procedures yearly. Nevertheless, the influence of their small experience in lymphatic mapping in the retroperitoneal space on the high negative sentinel node detection rate cannot be excluded. Finally, pancreatic lymphatic drainage is complex. Studies on staging of pancreatic head cancer revealed lymphatic routes draining to the celiac axis and superior mesenteric artery nodes. Some smaller branches may drain directly to paraaortic nodes and then to the thoracic duct [19], which are not visualized in irresectable tumors. Moreover, some authors indicate that pancreatic drainage may accompany multidirectional venous drainage with the absence of a solitary sentinel lymph node, similarly to lung cancer [20].

Despite the fact that our study failed to detect the accurate pattern of lymph flow from primary tumor of the pancreatic corpus, some interesting observations of lymphatic drainage should be noted. In one case two routes of lymphatic drainage were observed: a group of inferior drainage downwards into the superior mesenteric artery (group 14a) without an identified blue-dyed node, and a group of superior drainage into a solitary blue lymph node of the celiac axis (group 9) (Figs. 1, 2). In another study on lymphatic mapping in upper gastrointestinal cancers, usually two to three metastatic sentinel nodes in this station were blue-dyed. That highlights their important role in dissemination of gastrointestinal cancer cells within lymphatic channels [14]. Another interesting case was a solitary lymphatic route leading to a blue lymph node next to the left gastric artery (group 7), exceeding the usual lymphadenectomy area in pancreatic corpus cancers, which includes celiac plexus nodes (group 9), spleen artery and hilum nodes (group 10 and 11) and lymph nodes of the lower border of the pancreas (group 18). This confirms existence of the skip metastases phenomenon in pancreatic corpus cancer and proves their unpredictable occurrence. Nevertheless, the clinical implications of these observations will still be limited, until survival benefits of extended lymphadenectomy in patients with pancreatic cancer and metastatic lymph nodes are proved [21].

In conclusion, due to delayed detection of pancreatic body tumors, with resectability rate less than $10 \%$, feasibility of sentinel node navigation is considerably restricted. In the authors' opinion, further studies on lymphatic mapping in

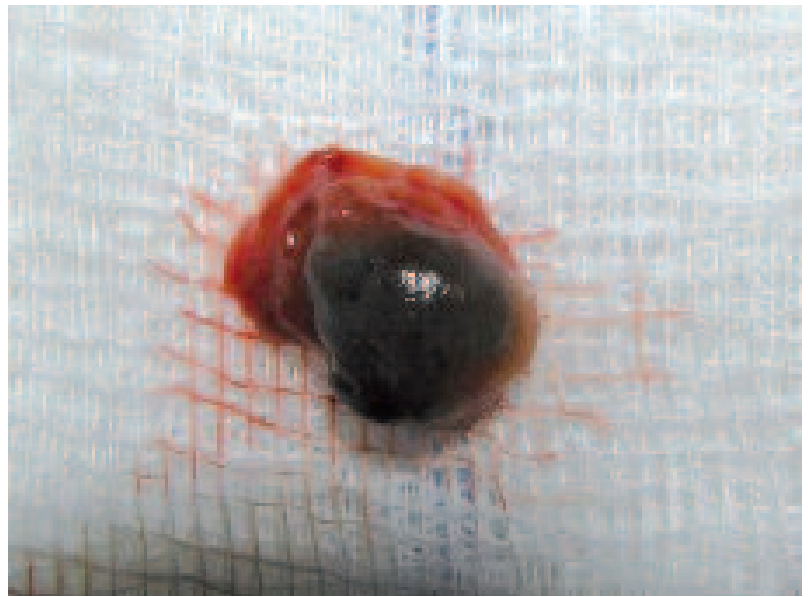

Fig. 2. Blue-dyed sentinel lymph node

a selected group of early stage tumors (smaller than $2 \mathrm{~cm}$ ) with the use of optimized newer markers may define the exact lymphatic drainage pattern from the pancreas. If confirmed by future studies, sentinel node biopsy might be suitable for patients who are preoperatively diagnosed with early pancreatic body cancer and minimally invasive surgery including laparoscopic distal pancreatectomy with personalized lymphadenectomy could be safely performed.

\section{References}

1. Loos M, Kleeff J, Friess H, Büchler MW. Surgical treatment of pancreatic cancer. Ann N Y Acad Sci 2008; 1138: 169-180.

2. Adams RB, Allen PJ. Surgical treatment of resectable and borderline resectable pancreatic cancer: expert consensus statement by Evans et al. Ann Surg Oncol 2009; 16: 1745-1750.

3. Alexakis N, Halloran C, Raraty M, Ghaneh P, Sutton R, Neoptolemos JP. Current standards of surgery for pancreatic cancer. Br J Surg 2004; 91: 1410-1427.

4. Morton DL, Wen DR, Wong JH, Economou JS, Cagle LA, Storm FK, Foshag LJ, Cochran AJ. Technical details of intraoperative lymphatic mapping for early stage melanoma. Arch Surg 1992; 127: 392-399.

5. Ferwerda CC, Statius Muller MG, Meijer S. The sentinel node concept in melanoma and breast cancer: relevancy and therapeutic consequences. Acta Chir Belg 2000; 100: 243-246.

6. Giuliano AE, Dale PS, Turner RR, Morton DL, Evans SW, Krasne DL. Improved axillary staging of breast cancer with sentinel lymphadenectomy. Ann Surg 1995; 222: 394-399.

7. Aikou T, Higashi H, Natsugoe S, Hokita S, Baba M, Tako S. Can sentinel node navigation surgery reduce the extent of lymph node dissection in gastric cancer? Ann Surg Oncol 2001; 8: 90-93.

8. Japanese Gastric Cancer Association. Japanese Classification of Gastric Carcinoma. 2nd English Edition - Gastric Cancer, 1998; 1: 10-24.

9. Pavlidis TE, Pavlidis ET, Sakantamis AK. Current opinion on lymphadenectomy in pancreatic cancer surgery. Hepatobiliary Pancreat Dis Int 2011; 10: 21-25.

10. Simsa J, Hoch J, Leffler J, Schwarz J, Pospisil R, Vajtrova R. Sentinel node biopsy in gastric cancer: preliminary results. Acta Chir Belg 2003; 103: 270-273.

11. Murawa D, Filas V, Breborowicz J, Spychała A, Dworzecka K, Murawa $P$. Evaluation of the sentinel node biopsy in colorectal carcinoma including the results of immunohistochemical examinations. Acta Chir Belg 2007; 107: 45-48.

12. Ohta T, Kitagawa H, Kayahara M, et al. Sentinel lymph node navigation surgery for pancreatic head cancers. Oncol Rep 2003; 10: 315-319.

13. Kocher HM, Sohail M, Benjamin IS, Patel AG. Technical limitations of lymph node mapping in pancreatic cancer. Eur J Surg Oncol 2007; 33: 887-891. 
14. Park SS, Ryu JS, Min BW, Kim WB, Kim SJ, Kim CS, Mok YJ. Impact of skip metastasis in gastric cancer. ANZ J Surg 2005; 75: 645-649.

15. Saha S, Bilchik A, Wiese D, et al. Ultrastaging of colorectal cancer by sentinel lymph node mapping technique: a multicenter trial. Ann Surg Oncol 2001; 8: 94-98.

16. Tsioulias GJ, Wood TF, Morton DL, Bilchik AJ. Lymphatic mapping and focused analysis of sentinel lymph nodes upstage gastrointestinal neoplasms. Arch Surg 2000; 135: 926-932.

17. Nano MT, Kollias J, Farshid G, Gill PG, Bochner M. Clinical impact of false-negative sentinel node biopsy in primary breast cancer. Br I Surg 2002; 89: 1430-1434.

18. Hutchinson JR, Chagpar AB, Scoggins CR, et al. Surgeon and community factors affecting breast cancer sentinel lymph node biopsy. Am J Surg 2005; 190: 903-906.

19. Kitagawa H, Ohta T, Makino I, et al. Carcinomas of the ventral and dorsal pancreas exhibit different patterns of lymphatic spread. Front Biosci 2008; 13: 2728-2735.

20. Rzyman W, Hagen OM, Dziadziuszko R, et al. Blue-dye intraoperative sentinel lymph node mapping in early non-small cell lung cancer. Eur I Surg Oncol 2006; 32: 462-465.

21. Yeo CJ, Cameron JL, Lillemoe KD, et al. Pancreaticoduodenectomy with or without distal gastrectomy and extended retroperitoneal lym phadenectomy for periampullary adenocarcinoma. Part 2: ran domized controlled trial evaluating survival, morbidity, and mortality. Ann Surg 2002; 236: 355-366

\section{Address for correspondence}

\section{Adam Durczyński}

Department of General and Transplant Surgery

Medical University of Lodz, Barlicki University Hospital

Kopcińskiego 22

90-153 Łódź, Poland

tel. +48426791091

e-mail: A.Durczynski@interia.pl

Submitted: $\quad$ 18.01.2012

Accepted: $\quad 11.05 .2012$ 